DOI 10.4171/JEMS/412

Stefan Friedl · Stefano Vidussi

\title{
A vanishing theorem for twisted Alexander polynomials with applications to symplectic 4-manifolds
}

Received December 23, 2011 and in revised form June 25, 2012

\begin{abstract}
We show that given any 3-manifold $N$ and any non-fibered class in $H^{1}(N ; \mathbb{Z})$ there exists a representation such that the corresponding twisted Alexander polynomial is zero. We obtain this result by extending earlier work of ours and by combining this with recent results of Agol and Wise on separability of 3-manifold groups. This result allows us to completely classify symplectic 4-manifolds with a free circle action, and to determine their symplectic cones.
\end{abstract}

\section{Introduction and main results}

A 3-manifold pair is a pair $(N, \phi)$ where $N$ is a compact, orientable, connected 3-manifold with toroidal or empty boundary, and $\phi \in H^{1}(N ; \mathbb{Z})=\operatorname{Hom}\left(\pi_{1}(N), \mathbb{Z}\right)$ is a nontrivial class. We say that a 3-manifold pair $(N, \phi)$ fibers over $S^{1}$ if there exists a fibration $p: N \rightarrow S^{1}$ such that the induced map $p_{*}: \pi_{1}(N) \rightarrow \pi_{1}\left(S^{1}\right)=\mathbb{Z}$ coincides with $\phi$. We refer to such $\phi$ as a fibered class.

Given a 3-manifold pair $(N, \phi)$ and an epimorphism $\alpha: \pi_{1}(N) \rightarrow G$ onto a finite group we can consider the twisted Alexander polynomial $\Delta_{N, \phi}^{\alpha} \in \mathbb{Z}\left[t^{ \pm 1}\right]$, whose definition is summarized in Section 2. It is well known that the twisted Alexander polynomials of a fibered class $\phi \in H^{1}(N)$ are monic and that their degrees are determined by the Thurston norm. In [FV11a] the authors showed that this condition is in fact sufficient to determine fiberedness. More precisely, if a nontrivial class $\phi \in H^{1}(N)$ is not fibered, then we proved in [FV11a] that there exists a twisted Alexander polynomial $\Delta_{N, \phi}^{\alpha}$ that fails to be monic or to have correct degree. We refer to Theorem 3.1 for the exact statement.

In previous work (see [FV08b]) the authors discussed how a stronger result, namely the vanishing of some twisted Alexander polynomial $\Delta_{N, \phi}^{\alpha}$, would follow assuming appropriate separability conditions for the fundamental group of $N$. In this paper, building on the techniques of [FV08b] supplemented with ideas of Wilton-Zalesskii [WZ10], and using new results on separability for 3-manifolds groups due to Agol and Wise we extend the vanishing result to all 3-manifolds. More precisely, we have the following

Theorem 1.1. Let $(N, \phi)$ be a 3-manifold pair. If $\phi \in H^{1}(N)$ is nonfibered, there exists an epimorphism $\alpha: \pi_{1}(N) \rightarrow G$ onto a finite group $G$ such that

$$
\Delta_{N, \phi}^{\alpha}=0 \text {. }
$$

S. Friedl: Mathematisches Institut, Universität zu Köln, Germany; e-mail: sfriedl@gmail.com S. Vidussi: Department of Mathematics, University of California, Riverside, CA 92521, USA; e-mail: svidussi@math.ucr.edu 
In brief, the strategy to prove Theorem 1.1 is the following. The techniques of [FV08b] allow one to prove a vanishing result under certain subgroup separability properties for the fundamental group of $N$. Recall that a group $\pi$ is called locally extended residually finite (LERF for short, or subgroup separable) if for any finitely generated subgroup $A \subset \pi$ and any $g \in \pi \backslash A$ there exists an epimorphism $\alpha: \pi \rightarrow G$ to a finite group $G$ such that $\alpha(g) \notin$ $\alpha(A)$. Using work of Wilton and Zalesskii [WZ10], we will reduce the separability condition to the hyperbolic pieces of $N$. It has been a longstanding conjecture that fundamental groups of hyperbolic 3-manifolds are LERF. Recently Dani Wise has made remarkable progress towards an affirmative answer. The following theorem combines the statements of Corollaries 14.3 and 14.16 and Theorem 16.28 of Wise [Wi12]. (We refer to [Wi09, Wi11, Wi12] and [AFW12] for background material, definitions and further information.)

Theorem 1.2 (Wise). If $N$ is either a closed hyperbolic 3-manifold which admits a geometrically finite surface or if $N$ is a hyperbolic 3-manifold with nontrivial boundary, then $\pi_{1}(N)$ is LERF.

In Section 4 we will show how this result can be used to complete the proof of Theorem 1.1. We want to add that Agol [Ag12] has made further progress in this direction, showing that the fundamental groups of any closed hyperbolic 3-manifold is LERF. (This implies as well, by work of Manning and Martínez-Pedroza [MMP10, Proposition 5.1] that the fundamental group of any hyperbolic 3-manifold with toroidal boundary is LERF.)

We now discuss the applications of Theorem 1.1. In Section 5 we will see that the combination of Theorem 1.1 with work of Goda and Pajitnov [GP05] implies a result on Morse-Novikov numbers of multiples of a given knot. Furthermore, we will see that the combination of Theorem 1.1 with work of Silver and Williams [SW09b] gives rise to a fibering criterion in terms of the number of finite covers of the $\phi$-cover of $N$. Arguably, however, the most interesting application of Theorem 1.1 is contained in Section 6, and regards the study of closed 4-manifolds with a free circle action which admit a symplectic structure. The main result of Section 6 is then the proof of the '(1) implies (3)' part of the following theorem:

Theorem 1.3. Let $N$ be a closed 3-manifold and let $p: M \rightarrow N$ be an $S^{1}$-bundle over $N$. Denote by $p_{*}: H^{2}(M ; \mathbb{R}) \rightarrow H^{1}(N ; \mathbb{R})$ the map given by integration along the fiber. Let $\psi \in H^{2}(M ; \mathbb{R})$. Then the following are equivalent:

(1) $\psi$ can be represented by a symplectic structure,

(2) $\psi$ can be represented by a symplectic structure that is $S^{1}$-invariant,

(3) $\psi^{2}>0$ and $\phi=p_{*}(\psi) \in H^{1}(N ; \mathbb{R})$ lies in the open cone on a fibered face of the Thurston norm ball of $N$.

(See Section 6 for details on the other implications.)

The implication '(1) implies (3)' was already shown to hold in the following cases:

(1) for reducible 3-manifolds by McCarthy [McC01],

(2) if $N$ has vanishing Thurston norm by Bowden [Bow09] and [FV08c], or if $N$ is a graph manifold, [FV08c], 
(3) if the canonical class of the symplectic structure is trivial, [FV11c],

(4) if $M$ is the trivial $S^{1}$-bundle over $N$, i.e. the case that $M=S^{1} \times N$, see [FV11a] for details.

Remark. (1) This paper can be viewed as the (presumably) last paper in a long sequence of papers [FV08a, FV08b, FV08c, FV11a, FV11b, FV11c] by the authors on twisted Alexander polynomials, fibered 3-manifolds and symplectic structures.

(2) Some steps in the proof of Theorem 1.3 (notably Propositions 6.2 and 6.3) already appeared in an unpublished manuscript by the authors (see [FV08c]).

(3) Bowden [Bow12] used Theorem 1.3 to determine which 4-manifolds with a fixed point free circle action are symplectic.

Convention. Unless it says specifically otherwise, all groups are assumed to be finitely generated, all manifolds are assumed to be orientable, connected and compact, and all 3-manifolds are assumed to have empty or toroidal boundary.

\section{Definition of twisted Alexander polynomials}

In this section we quickly recall the definition of twisted Alexander polynomials. This invariant was initially introduced by Lin [Li01], Wada [Wa94] and Kirk-Livingston [KL99]. We refer to [FV10a] for a detailed presentation.

Let $X$ be a finite CW complex, let $\phi \in H^{1}(X ; \mathbb{Z})=\operatorname{Hom}\left(\pi_{1}(X), \mathbb{Z}\right)$ and let $\alpha: \pi_{1}(X)$ $\rightarrow \operatorname{GL}(n, R)$ be a representation over a Noetherian unique factorization domain $R$. In our applications we will take $R=\mathbb{Z}$ or $R=\mathbb{Q}$. We can now define a left $\mathbb{Z}\left[\pi_{1}(X)\right]$-module structure on $R^{n} \otimes_{\mathbb{Z}} \mathbb{Z}\left[t^{ \pm 1}\right]=: R^{n}\left[t^{ \pm 1}\right]$ as follows:

$$
g \cdot(v \otimes p):=(\alpha(g) \cdot v) \otimes\left(t^{\phi(g)} p\right),
$$

where $g \in \pi_{1}(X)$ and $v \otimes p \in R^{n} \otimes_{\mathbb{Z}} \mathbb{Z}\left[t^{ \pm 1}\right]=R^{n}\left[t^{ \pm 1}\right]$. Put differently, we get a representation $\alpha \otimes \phi: \pi_{1}(X) \rightarrow \mathrm{GL}\left(n, R\left[t^{ \pm 1}\right]\right)$.

Denote by $\widetilde{X}$ the universal cover of $X$. Letting $\pi=\pi_{1}(X)$, we use the representation $\alpha \otimes \phi$ to regard $R^{n}\left[t^{ \pm 1}\right]$ as a left $\mathbb{Z}[\pi]$-module. The chain complex $C_{*}(\tilde{X})$ is also a left $\mathbb{Z}[\pi]$-module via deck transformations. Using the natural involution $g \mapsto g^{-1}$ on the group ring $\mathbb{Z}[\pi]$ we can view $C_{*}(\tilde{X})$ as a right $\mathbb{Z}[\pi]$-module. We can therefore consider the tensor products

$$
C_{*}^{\phi \otimes \alpha}\left(X ; R^{n}\left[t^{ \pm 1}\right]\right):=C_{*}(\tilde{X}) \otimes_{\mathbb{Z}\left[\pi_{1}(X)\right]} R^{n}\left[t^{ \pm 1}\right],
$$

which form a complex of $R\left[t^{ \pm 1}\right]$-modules. We then consider the $R\left[t^{ \pm 1}\right]$-modules

$$
H_{*}^{\phi \otimes \alpha}\left(X ; R^{n}\left[t^{ \pm 1}\right]\right):=H_{*}\left(C_{*}^{\phi \otimes \alpha}\left(X ; R^{n}\left[t^{ \pm 1}\right]\right)\right) .
$$

If $\alpha$ and $\phi$ are understood we will drop them from the notation. Since $X$ is compact and since $R\left[t^{ \pm 1}\right]$ is Noetherian these modules are finitely presented over $R\left[t^{ \pm 1}\right]$. We now define the twisted Alexander polynomial of $(X, \phi, \alpha)$ to be the order of $H_{1}\left(X ; R^{n}\left[t^{ \pm 1}\right]\right)$ 
(see [FV10a] and [Tu01, Section 4] for details). We will denote it as $\Delta_{X, \phi}^{\alpha} \in R\left[t^{ \pm 1}\right]$. Note that $\Delta_{X, \phi}^{\alpha} \in \mathbb{Z}\left[t^{ \pm 1}\right]$ is well defined up to multiplication by a unit in $R\left[t^{ \pm 1}\right]$. We adopt the convention that we drop $\alpha$ from the notation if $\alpha$ is the trivial representation to $\operatorname{GL}(1, \mathbb{Z})$.

If $\alpha: \pi_{1}(N) \rightarrow G$ is a homomorphism to a finite group $G$, then we get the regular representation $\pi_{1}(N) \rightarrow G \rightarrow \operatorname{Aut}_{\mathbb{Z}}(\mathbb{Z}[G])$, where the second map is given by left multiplication. We can identify $\operatorname{Aut}_{\mathbb{Z}}(\mathbb{Z}[G])$ with $\operatorname{GL}(|G|, \mathbb{Z})$ and we obtain the corresponding twisted Alexander polynomial $\Delta_{N, \phi}^{\alpha}$. We will sometimes write $H_{*}\left(X ; \mathbb{Z}[G]\left[t^{ \pm 1}\right]\right)$ instead of $H_{*}\left(X ; \mathbb{Z}^{|G|}\left[t^{ \pm 1}\right]\right)$.

The following lemma is well known (see e.g. [Tu01, Remark 4.5]).

Lemma 2.1. Let $(N, \phi)$ be a 3-manifold pair and let $\alpha: \pi_{1}(N) \rightarrow G$ be a homomorphism to a finite group. Then $\Delta_{N, \phi}^{\alpha} \neq 0$ if and only if $H_{1}\left(N ; \mathbb{Z}[G]\left[t^{ \pm 1}\right]\right)$ is $\mathbb{Z}\left[t^{ \pm 1}\right]$-torsion.

Later, we will need the following well known lemma.

Lemma 2.2. Let $(N, \phi)$ be a 3-manifold pair. Let $\alpha: \pi_{1}(N) \rightarrow G$ and $\beta: \pi_{1}(N) \rightarrow H$ be homomorphisms to finite groups such that $\operatorname{Ker}(\alpha) \subset \operatorname{Ker}(\beta)$. Then there exists $p$ in $\mathbb{Q}\left[t^{ \pm 1}\right]$ such that

$$
\Delta_{N, \phi}^{\alpha}=\Delta_{N, \phi}^{\beta} \cdot p \in \mathbb{Q}\left[t^{ \pm 1}\right]
$$

In particular, if $\Delta_{N, \phi}^{\beta}=0$, then $\Delta_{N, \phi}^{\alpha}=0$.

Proof. We denote by $\alpha$ also the regular representation $\pi_{1}(N) \rightarrow \operatorname{Aut}_{\mathbb{Q}}(\mathbb{Q}[G])$, and similarly we denote by $\beta$ the regular representation $\pi_{1}(N) \rightarrow \operatorname{Aut}_{\mathbb{Q}}(\mathbb{Q}[H])$. Note that the assumption $\operatorname{Ker}(\alpha) \subset \operatorname{Ker}(\beta)$ implies there exists an epimorphism $\gamma: G \rightarrow H$ such that $\beta=\gamma \circ \alpha$. Note that $\gamma$ endows $\mathbb{Q}[H]$ with the structure of a left $\mathbb{Q}[G]$-module. It follows from Maschke's theorem (see [La02, Theorem XVIII.1.2]) that there exists a left $\mathbb{Q}[G]$-module $P$ and an isomorphism of left $\mathbb{Q}[G]$-modules

$$
\mathbb{Q}[G] \cong \mathbb{Q}[H] \oplus P .
$$

We now denote by $\rho$ the representation $\pi_{1}(N) \stackrel{\alpha}{\rightarrow} G \rightarrow \operatorname{Aut}(P) \cong \mathrm{GL}(\operatorname{dim}(P), \mathbb{Q})$. It now follows from the definitions that

$$
\Delta_{N, \phi}^{\alpha}=\Delta_{N, \phi}^{\beta} \cdot \Delta_{N, \phi}^{\rho}
$$

\section{Twisted Alexander polynomials and fibered 3-manifolds}

Let $(N, \phi)$ be a 3-manifold pair. We denote by $\|\phi\|_{T}$ the Thurston norm of a class $\phi \epsilon$ $H^{1}(N ; \mathbb{Z})$; we refer to [Th86] for details. We say that $p(t) \in \mathbb{Z}\left[t^{ \pm 1}\right]$ is monic if its top coefficient equals \pm 1 , and given a nonzero polynomial $p(t) \in \mathbb{Z}\left[t^{ \pm 1}\right]$ with $p=$ $\sum_{i=k}^{l} a_{i} t^{i}, a_{k} \neq 0, a_{l} \neq 0$, we define $\operatorname{deg}(p)=l-k$.

It is known that twisted Alexander polynomials give complete fibering obstructions. In fact the following theorem holds: 
Theorem 3.1. Let $(N, \phi)$ be a 3-manifold pair where $N \neq S^{1} \times S^{2}, S^{1} \times D^{2}$. Then $(N, \phi)$ is fibered if and only if for any epimorphism $\alpha: \pi_{1}(N) \rightarrow G$ onto a finite group the twisted Alexander polynomial $\Delta_{N, \phi}^{\alpha} \in \mathbb{Z}\left[t^{ \pm 1}\right]$ is monic and

$$
\operatorname{deg}\left(\Delta_{N, \phi}^{\alpha}\right)=|G| \cdot\|\phi\|_{T}+\left(1+b_{3}(N)\right) \operatorname{div} \phi_{\alpha},
$$

where $\phi_{\alpha}$ denotes the restriction of $\phi: \pi_{1}(N) \rightarrow \mathbb{Z}$ to $\operatorname{Ker}(\alpha)$, and where we denote by $\operatorname{div} \phi_{\alpha} \in \mathbb{N}$ the divisibility of $\phi_{\alpha}$, i.e.

$$
\operatorname{div} \phi_{\alpha}=\max \left\{n \in \mathbb{N} \mid \phi_{\alpha}=n \psi \text { for some } \psi: \operatorname{Ker}(\alpha) \rightarrow \mathbb{Z}\right\} .
$$

The 'only if' direction has been shown at various levels of generality by Cha [Ch03], Kitano and Morifuji [KM05], Goda, Kitano and Morifuji [GKM05], Pajitnov [Pa07], Kitayama [Kiy07], [FK06] and [FV10a, Theorem 6.2]. The 'if' direction is the main result of [FV11a]. We also refer to [FV11b] for a more leisurely approach to the proof of the 'if' direction.

\section{The proof of Theorem 1.1}

In this section we will prove Theorem 1.1. The approach we follow is the one we used in [FV08b] to cover the case of a 3-manifold with certain subgroup separability properties, adding as new ingredient the work of Wilton and Zalesskii [WZ10] (which builds in turn on work of Hamilton [Ham01]), to cover the case where the 3-manifold has a nontrivial Jaco-Shalen-Johannson (JSJ) decomposition (see the original work of Jaco-Shalen [JS79] or Johannson [Jo79] or also [AFW12] for details). We start with the following.

Lemma 4.1. Let $N$ be an irreducible 3-manifold with JSJ pieces $N_{v}, v \in V$. Let $\alpha_{v}: \pi_{1}\left(N_{v}\right) \rightarrow G_{v}, v \in V$, be homomorphisms to finite groups. Then there exists an epimorphism $\beta: \pi_{1}(N) \rightarrow G$ to a finite group such that $\operatorname{Ker}(\beta) \cap \pi_{1}\left(N_{v}\right) \subset \operatorname{Ker}\left(\alpha_{v}\right)$ for all $v \in V$.

Proof. We write $K_{v}:=\operatorname{Ker}\left(\alpha_{v}\right), v \in V$. Evidently there exists an $n \in \mathbb{N}$ with the following property: for each $v \in V$ and each boundary torus $T$ of $N_{v}$ we have

$$
n \cdot \pi_{1}(T) \subset \pi_{1}(T) \cap K_{v} .
$$

(Here $n \cdot \pi_{1}(T)$ denotes the unique subgroup of $\pi_{1}(T) \cong \mathbb{Z}^{2}$ of index $n^{2}$.) By [WZ10, Theorem 3.2] (which relies strongly on Lemmas 5 and 6 of [Ham01]) there exists an $m \in \mathbb{N}$ and finite index normal subgroups $L_{v} \subset \pi_{1}\left(N_{v}\right), v \in V$, such that for each $v \in V$ and each boundary torus $T$ of $N_{v}$ we have

$$
n m \cdot \pi_{1}(T)=\pi_{1}(T) \cap L_{v} .
$$

We now define $M_{v}=K_{v} \cap L_{v}, v \in V$. Note that for each JSJ torus $T$ and two adjacent JSJ pieces the intersection of the corresponding $M$-groups is $n m \cdot \pi_{1}(T)$. It now follows from a standard argument (see e.g. [WZ10, Proof of Theorem 3.7]) that there exists a finite index normal subgroup $M$ of $\pi_{1}(N)$ such that $M \cap \pi_{1}\left(N_{v}\right) \subset M_{v}$ for any $v \in V$. Clearly the epimorphism $\pi_{1}(N) \rightarrow \pi_{1}(N) / M$ has the desired properties.

For the reader's convenience we recall the statement of Theorem 1.1. 
Theorem 4.2. Let $(N, \phi)$ be a 3-manifold pair. Then if $\phi \in H^{1}(N)$ is nonfibered, there exists an epimorphism $\alpha: \pi_{1}(N) \rightarrow G$ onto a finite group $G$ such that

$$
\Delta_{N, \phi}^{\alpha}=0 .
$$

Remark. As mentioned above, a proof of this theorem appears in [FV08b] for a 3-manifold $N$ in the following two cases:

(1) if the subgroups carried by Thurston norm minimizing surfaces are separable, or

(2) if $N$ is a graph manifold.

Recent work of Przytycki and Wise [PW11, Theorem 1.1] shows that the separability condition (1) is in fact satisfied for all graph manifolds, which gives an alternative proof for (2).

Proof of Theorem 4.2. If $N$ is reducible, the statement is proven to hold in [FV11a, Lemma 7.1]. Hence we restrict ourselves to the case where $N$ is irreducible. We denote by $\left\{N_{v}\right\}_{v \in V}$ the set of JSJ components of $N$ and by $\left\{T_{e}\right\}_{e \in E}$ the set of JSJ tori in the JSJ decomposition of $N$. Let $\phi \in H^{1}(N ; \mathbb{Z})$ be a nonfibered class. If the restriction of $\phi$ to one of the JSJ tori is trivial, then it follows from [FV08b, Theorem 5.2] that there exists an epimorphism $\alpha: \pi_{1}(N) \rightarrow G$ onto a finite group $G$ such that $\Delta_{N, \phi}^{\alpha}=0$. We will henceforth assume that the restriction of $\phi$ to all JSJ tori is nontrivial.

Given $v \in V$ we denote by $\phi_{v}$ the restriction of $\phi$ to $N_{v}$. Since $\phi$ is nonfibered it follows from [EN85, Theorem 4.2] that there exists a $w \in V$ such that $\left(N_{w}, \phi_{w}\right)$ is not fibered. If $N_{w}=N$ is hyperbolic and closed (i.e. the JSJ decomposition is trivial), as we assume that $(N, \phi)$ is not fibered, $N$ admits an incompressible surface $\Sigma$ which does not lift to a fiber in any finite cover. By a result of Bonahon and Thurston (see [Bon86]) the surface $\Sigma$ is a geometrically finite surface. It then follows from Theorem 1.2 that $\pi_{1}(N)$ is LERF. If $N_{w}$ is hyperbolic and has nontrivial boundary, Theorem 1.2 guarantees again that $\pi_{1}\left(N_{w}\right)$ is LERF. The same holds if $N_{w}$ is Seifert fibered, by Scott's theorem (see [Sc78]).

By [FV08b, Theorem 4.2] there exists in either case an epimorphism $\alpha_{w}: \pi_{1}\left(N_{w}\right) \rightarrow$ $G_{w}$ onto a finite group $G_{w}$ such that $\Delta_{N_{w}, \phi_{w}}^{\alpha_{w}}=0$. By Lemma 4.1 above there exists a homomorphism $\beta: \pi_{1}(N) \rightarrow G$ to a finite group such that $\operatorname{Ker}(\beta) \cap \pi_{1}\left(N_{w}\right)=\operatorname{Ker}\left(\beta_{w}\right) \subset$ $\operatorname{Ker}\left(\alpha_{w}\right)$. (Here, given $v \in V$ we denote by $\beta_{v}$ the restriction of $\beta$ to $N_{v}$.) By Lemma 2.2 we also have $\Delta_{N_{w}, \phi_{w}}^{\beta_{w}}=0$.

Now, there exists a Mayer-Vietoris type long exact sequence of twisted homology groups:

$\cdots \rightarrow \bigoplus_{e \in E} H_{i}\left(T_{e} ; \mathbb{Z}[G]\left[t^{ \pm 1}\right]\right) \rightarrow \bigoplus_{v \in V} H_{i}\left(N_{v} ; \mathbb{Z}[G]\left[t^{ \pm 1}\right]\right) \rightarrow H_{i}\left(N ; \mathbb{Z}[G]\left[t^{ \pm 1}\right]\right) \rightarrow \cdots$

Recall that we assumed that the restriction of $\phi$ to $T_{e}$ is nontrivial for each $e$. It follows from a straightforward calculation (see e.g. [KL99, p. 644]) that $H_{i}\left(T_{e} ; \mathbb{Z}[G]\left[t^{ \pm 1}\right]\right)$ is $\mathbb{Z}\left[t^{ \pm 1}\right]$-torsion for each $i$ and each $e \in E$. As $\Delta_{N_{w}, \phi_{w}}^{\beta_{v}}=0$, Lemma 2.1 implies that $H_{1}\left(N_{w} ; \mathbb{Z}[G]\left[t^{ \pm 1}\right]\right)$ is not $\mathbb{Z}\left[t^{ \pm 1}\right]$-torsion. But then it follows from the above exact sequence that $H_{1}\left(N ; \mathbb{Z}[G]\left[t^{ \pm 1}\right]\right)$ is not $\mathbb{Z}\left[t^{ \pm 1}\right]$-torsion either, i.e. $\Delta_{N, \phi}^{\beta}=0$. 


\section{Applications to 3-manifold topology}

Let $K \subset S^{3}$ be a knot. We denote by $X_{K}:=S^{3} \backslash \nu K$ the exterior of $K$. A regular Morse function on $X_{K}$ is a function $f: X_{K} \rightarrow S^{1}$ such that all singularities are nondegenerate and which restricts on the boundary of $X_{K}$ to a fibration with connected fiber. Given a Morse map $f$ we denote by $m_{i}(f)$ the number of critical points of index $i$. A regular Morse function $f: X_{K} \rightarrow S^{1}$ is called minimal if $m_{i}(f) \leq m_{i}(g)$ for any regular Morse map $g$ and any $i$. It is shown by Pajitnov, Rudolph and Weber [PRW02] that any knot admits a minimal regular Morse function. Its number of critical points is called the MorseNovikov number of $K$ and denoted by $\mathcal{M N}(K)$. Note that $K$ is fibered if and only if $\mathcal{M N}(K)=0$. It is known that $\mathcal{M N}\left(K_{1} \# K_{2}\right) \leq \mathcal{M N}\left(K_{1}\right)+\mathcal{M N}\left(K_{2}\right)$ (see [PRW02, Proposition 6.2]), but it is not known whether equality holds. It is not even known whether $\mathcal{M N}(n \cdot K)=n \cdot \mathcal{M N}(K)$. The following theorem can be viewed as evidence to an affirmative answer for the latter question.

Theorem 5.1. Let $K \subset S^{3}$ be a nonfibered knot. Then there exists a $\lambda>0$ such that

$$
\mathcal{M N}(n \cdot K) \geq n \cdot \lambda \text {. }
$$

This theorem is an immediate consequence of Theorem 1.1 and results of Goda and Pajitnov [GP05, Theorem 4.2 and Corollary 4.6]. The statement is similar in spirit to a result by Pajitnov (see [Pa10, Proposition 4.2]) on the tunnel number of multiples of a given knot.

Now let $(N, \phi)$ be a fibered 3-manifold pair. In that case $\operatorname{Ker}\left(\phi: \pi_{1}(N) \rightarrow \mathbb{Z}\right)$ is the fundamental group of a surface, in particular it is a finitely generated group, and it follows that the $\phi$-cover of $N$ admits only countably many finite covers. The next theorem, which follows by combining Theorem 1.1 with work of Silver and Williams (see [SW09a, SW09b]), says that the converse to the above statement holds true.

Theorem 5.2. Let $N$ be a 3-manifold and let $\phi \in H^{1}(N)=\operatorname{Hom}\left(\pi_{1}(N), \mathbb{Z}\right)$. If $(N, \phi)$ does not fiber over $S^{1}$, then the $\phi$-cover of $N$ admits uncountably many finite covers.

For knot exteriors this result is an immediate consequence of Theorem 1.1 and a result of Silver and Williams [SW09b, Theorem 3.4] for knots (see also [SW09a]). It is straightforward to verify that the argument by Silver and Williams carries over to the general case.

Note that Theorem 5.2 can be viewed as a significant strengthening of Stallings' fibering theorem (see [St62]), which says that a class $\phi \in H^{1}(N)=\operatorname{Hom}\left(\pi_{1}(N), \mathbb{Z}\right)$ is fibered if and only if $\operatorname{Ker}(\phi)$ is finitely generated.

\section{Symplectic 4-manifolds with a free circle action}

In this section we will prove Theorem 1.3.

\subsection{Preliminaries}

We start by recalling some elementary facts about the algebraic topology of a 4-manifold $M$ that carries a free circle action. The free circle action renders $M$ the to- 
tal space of a circle bundle $p: M \rightarrow N$ over the orbit space, with Euler class $e \in H^{2}(N)$. For the purpose of proving Theorem 1.3, we will see that we can limit the discussion to the case where the Euler class is nontorsion, so we will make this assumption for the rest of the subsection. The Gysin sequence reads

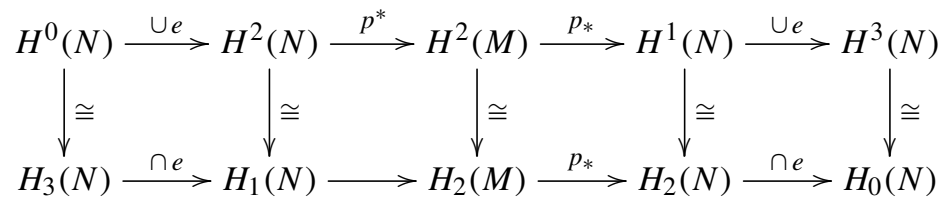

where $p_{*}: H^{2}(M) \rightarrow H^{1}(N)$ denotes integration along the fiber. In particular we have

$$
0 \rightarrow\langle e\rangle \rightarrow H^{2}(N) \stackrel{p^{*}}{\rightarrow} H^{2}(M) \stackrel{p_{*}}{\rightarrow} \operatorname{ker}(\cdot e) \rightarrow 0,
$$

where $\langle e\rangle$ is the cyclic subgroup of $H^{2}(N)$ generated by the Euler class and where $\operatorname{ker}(\cdot e)$ denotes the subgroup of elements in $H^{1}(N)$ whose pairing with the Euler class vanishes. As $e$ is nontorsion, it follows that $b_{2}(M)=2 b_{1}(N)-2$. It is not difficult to verify that $\operatorname{sign}(M)=0$, hence $b_{2}^{+}(M)=b_{1}(N)-1$.

Let $\alpha: \pi_{1}(N) \rightarrow G$ be a homomorphism to a finite group. We denote by $\pi: N_{G} \rightarrow N$ the regular $G$-cover of $N$. It is well known that $b_{1}\left(N_{G}\right) \geq b_{1}(N)$. If $\pi: M \rightarrow N$ is a circle bundle with Euler class $e \in H^{2}(N)$ then $\alpha$ determines a regular $G$-cover of $M$ that we will denote (with slight abuse of notation) $\pi: M_{G} \rightarrow M$. These covers are related by the commutative diagram

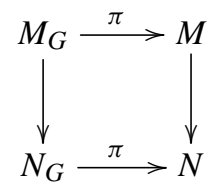

where the circle bundle $p_{G}: M_{G} \rightarrow N_{G}$ has Euler class $e_{G}=\pi^{*} e \in H^{2}\left(N_{G}\right)$ that is nontorsion as $e$ is.

\subsection{Seiberg-Witten theory for symplectic manifolds with a circle action}

In this section we will apply, for the class of manifolds we are studying, Taubes' nonvanishing theorem for Seiberg-Witten invariants of symplectic manifold to get a restriction on the class of orbit spaces of a free circle action over a symplectic 4-manifold. In order to do so, we need to understand the Seiberg-Witten invariants of $M$. Again, we will limit the discussion here to the case where the Euler class $e \in H^{2}(N)$ is not torsion. (The torsion case will be treated as a corollary of [FV11a].) The essential ingredient is the fact that the Seiberg-Witten invariants of $M$ are related to the Alexander polynomial of $N$. Baldridge proved the following result, that combines Corollaries 25 and 27 of [Ba03] (cf. also [Ba01]), to which we refer the reader for definitions and results for Seiberg-Witten theory in this set-up: 
Theorem 6.1 (Baldridge). Let $M$ be a 4-manifold admitting a free circle action with nontorsion Euler class $e \in H^{2}(N)$, where $N$ is the orbit space. Then the Seiberg-Witten invariant $S W_{M}(\kappa)$ of a class $\kappa=p^{*} \xi \in p^{*} H^{2}(N) \subset H^{2}(M)$ is given by the formula

$$
S W_{M}(\kappa)=\sum_{l \in \mathbb{Z}} S W_{N}(\xi+l e) \in \mathbb{Z},
$$

in particular when $b_{2}^{+}(M)=1$ it is independent of the chamber in which it was calculated. Moreover, if $b_{2}^{+}(M)>1$, these are the only basic classes.

As $H^{2}(N)$ acts freely and transitively on $\operatorname{spin}^{c}(N)$ (and similarly for $M$ ), we use the existence of a product $\operatorname{spin}^{c}$ structure on $N$ (respectively $M$ ) to henceforth identify the set of $\operatorname{spin}^{c}$ structures with $H^{2}(N)$ (respectively $H^{2}(M)$ ). Observe that, with this identification, the first Chern class of a $\operatorname{spin}^{c}$ structure on $N$ satisfies $c_{1}(\xi)=2 \xi \in H^{2}(N)$ (and similarly on $M$ ).

The Seiberg-Witten invariants of $N$ determine, via [MT96], the Alexander polynomial of $N$. Assuming that a manifold $M$ as above is symplectic, we will use Taubes' constraints on its Seiberg-Witten invariants and Baldridge's formula to get a constraint on the twisted Alexander polynomials of $N$. We start with a technical lemma.

Lemma 6.2. Let $(M, \omega)$ be a symplectic manifold admitting a free circle action with nontorsion Euler class $e \in H^{2}(N)$, where $N$ is the orbit space. Then the canonical class $K \in H^{2}(M)$ of the symplectic structure is the pull-back of a class $\zeta \in H^{2}(N)$, where $\zeta$ is well defined up to the addition of a multiple of $e$.

Proof. If $b_{2}^{+}(M)>1$ this is a straightforward consequence of Theorem 6.1, as the canonical class by [Ta94] is a basic class of $M$, hence must be the pull-back of a class of $H^{2}(N)$. The case of $b_{2}^{+}(M)=1$ can be similarly obtained by a careful analysis of the chamber structure of the Seiberg-Witten invariants for classes that are not pulled back from $N$, but it is possible to use a quicker argument. First, observe that as the 2-torsion of $H^{2}(M)$ is contained in $p^{*} H^{2}(N)$, a spin ${ }^{c}$ structure on $M$ is pull-back if and only if its first Chern class is pull-back. Next, starting from a closed curve in $N$ representing a suitable element of $H_{1}(N)$, we can identify a torus $T \subset M$ of self-intersection zero, representing the generator of a cyclic subgroup in the image of the map $H_{1}(N) \rightarrow H_{2}(M)$ in the homology Gysin sequence, that satisfies $\omega \cdot[T]>0$. Second we can assume, by [Liu96], that $K \cdot \omega \geq 0$. (Otherwise $M$ would be a rational or ruled surface; using classical invariants, the only possibility would be $M=S^{2} \times T^{2}$, but then $e=0$.) Also, as both signature and Euler characteristic of $M$ vanish, $K^{2}=2 \chi(M)+3 \sigma(M)=0$. Omitting the case of $K$ torsion, where the statement is immediate, we deduce that both $K$ and (the Poincaré dual of) $[T]$ lie in the closure of the forward positive cone in $H^{2}(M, \mathbb{R})$ determined by $\omega$. The light-cone lemma (see e.g. [Liu96]) asserts at this point that $K \cdot[T] \geq 0$. On the other hand, the adjunction inequality of $\mathrm{Li}$ and Liu (see [LL95]) gives $K \cdot[T] \leq 0$, hence $K \cdot[T]=0$. It now follows that $K$ is a multiple of $P D([T])$, in particular the pull-back of a class on $N$.

Let then $(M, \omega)$ be a symplectic manifold admitting a free circle action with orbit space $N$ and nontorsion Euler class $e \in H^{2}(N)$. As $[\omega]^{2}>0$ it follows from Section 6.1 that 
$[\omega] \notin p^{*}\left(H^{2}(N, \mathbb{R})\right)$, and in particular $p_{*}[\omega] \neq 0 \in H^{1}(N ; \mathbb{R})$. Using openness of the symplectic condition, we can assume that $[\omega] \in H^{2}(M ; \mathbb{R})$ lies in the rational lattice (identified with) $H^{2}(M ; \mathbb{Q})$. After suitably scaling $\omega$ by a rational number if needed, the class $p_{*}[\omega]$ is then (the image of) a primitive (in particular, nonzero) class in $H^{1}(N ; \mathbb{Z})$ that we denote by $\phi$.

We are in a position now to use equation (2) to obtain the following.

Proposition 6.3. Let $(M, \omega)$ be a symplectic manifold admitting a free circle action with nontorsion Euler class such that $\phi=p_{*}[\omega] \in H^{1}(N)$ is an integral class on the orbit space $N$. Then for all epimorphisms $\alpha: \pi_{1}(N) \rightarrow G$ to a finite group the twisted Alexander polynomial $\Delta_{N, \phi}^{\alpha} \in \mathbb{Z}\left[t^{ \pm 1}\right]$ is monic of Laurent degree

$$
\operatorname{deg} \Delta_{N, \phi}^{\alpha}=|G| \zeta \cdot \phi+2 \operatorname{div} \phi_{G}
$$

Here, $\zeta \in H^{2}(N)$ is a class whose pull-back to $M$ gives the canonical class of $M$. Furthermore $\phi_{G}$ denotes the restriction of $\phi: \pi_{1}(N) \rightarrow \mathbb{Z}$ to $\operatorname{Ker}(\alpha)$ and $\operatorname{div} \phi_{G}$ stands for the divisibility of $\phi_{G}$.

Proof. The proof follows by application of Taubes' results ([Ta94, Ta95]) on the SeibergWitten invariants of finite covers of $M$ to impose constraints on the twisted Alexander polynomials of $N$. We will first analyze the constraints on the ordinary 1-variable Alexander polynomial $\Delta_{N, \phi}$. By [FV08a, Theorem 3.5] we can write this polynomial as

$$
\Delta_{N, \phi}=\left(t^{\operatorname{div} \phi}-1\right)^{2} \cdot \sum_{g \in H} a_{g} t^{\phi(g)} \in \mathbb{Z}\left[t^{ \pm 1}\right]
$$

where $H$ is the maximal free abelian quotient of $\pi_{1}(N)$ and $\Delta_{N}=\sum_{g \in H} a_{g} \cdot g \in \mathbb{Z}[H]$ is the ordinary multivariable Alexander polynomial of $N$. By Meng and Taubes [MT96] the latter is related to the Seiberg-Witten invariants of $N$ via the formula

$$
\sum_{g \in H} a_{g} \cdot g= \pm \sum_{\xi \in H^{2}(N)} S W_{N}(\xi) \cdot f(\xi) \in \mathbb{Z}[H]
$$

where $f$ denotes the composition of Poincaré duality with the quotient map $f: H^{2}(N) \cong$ $H_{1}(N) \rightarrow H$. Using this formula, we can write

$$
\Delta_{N, \phi}= \pm\left(t^{\operatorname{div} \phi}-1\right)^{2} \sum_{\xi \in H^{2}(N)} S W_{N}(\xi) t^{\phi \cdot \xi} .
$$

We will now use (2) to write $\Delta_{N, \phi}$ in terms of the 4-dimensional Seiberg-Witten invariants of $M$. In order to do so, observe that for all classes $\xi \in H^{2}(N)$ we can write $\xi \cdot \phi=\xi \cdot p_{*} \omega=p^{*} \xi \cdot \omega=\kappa \cdot \omega$ where $\kappa=p^{*} \xi$. Grouping together the contributions of the 3-dimensional basic classes in terms of their image in $H^{2}(M)$, and using the fact that $(\xi+l e) \cdot \phi=\xi \cdot \phi$ and (2) we get

$$
\begin{aligned}
\Delta_{N, \phi} & = \pm\left(t^{\operatorname{div} \phi}-1\right)^{2} \sum_{\kappa \in p^{*} H^{2}(N)} \sum_{l} S W_{N}(\xi+l e) t^{\phi \cdot \xi} \\
& = \pm\left(t^{\operatorname{div} \phi}-1\right)^{2} \sum_{\kappa \in p^{*} H^{2}(N)} S W_{M}(\kappa) t^{\kappa \cdot \omega} .
\end{aligned}
$$


Taubes' constraints, applied to the symplectic manifold $(M, \omega)$, assert that the canonical $\operatorname{spin}^{c}$ structure $\kappa_{\omega}$, with first Chern class $c_{1}\left(\kappa_{\omega}\right)=-K=-p^{*} \zeta \in H^{2}(M)$, satisfies $S W_{M}\left(\kappa_{\omega}\right)=1$. Moreover, for all $\operatorname{spin}^{c}$ structure $\kappa$ with $S W_{M}(\kappa) \neq 0$, we have

$$
\kappa_{\omega} \cdot \omega \leq \kappa \cdot \omega
$$

with equality possible only for $\kappa=\kappa_{\omega}$. (When $b_{2}^{+}(M)=1$, this statement applies to the Seiberg-Witten invariants evaluated in Taubes' chamber, but as remarked in Theorem 6.1 this specification is not a concern in our situation.) It now follows that $\Delta_{N, \phi}$ is a monic polynomial, and remembering the symmetry of $S W_{N}$ (or $\Delta_{N, \phi}$ ), we see that its Laurent degree is $d=-2 \kappa_{\omega} \cdot \omega+2 \operatorname{div} \phi=K \cdot \omega+2 \operatorname{div} \phi=\zeta \cdot \phi+2 \operatorname{div} \phi$.

Consider now any symplectic 4-manifold $M$ satisfying the hypothesis of the statement. Take an epimorphism $\alpha: \pi_{1}(N) \rightarrow G$ and denote, as in Section 6.1, by $N_{G}$ and $M_{G}$ the associated $G$-covers of $N$ and $M$ respectively. We will bootstrap the constraint on the ordinary Alexander polynomials to the twisted Alexander polynomials associated to $\alpha: \pi_{1}(N) \rightarrow G$. As $(M, \omega)$ is symplectic, $M_{G}$ inherits a symplectic form $\omega_{G}:=\pi^{*} \omega$ with canonical class $K_{G}:=\pi^{*} K$, that is easily shown to satisfy the condition $\phi_{G}:=\left(p_{G}\right)_{*}\left[\omega_{G}\right]=\pi^{*} \phi \in H^{1}\left(N_{G}\right)$. We can therefore apply the results of the previous paragraph to the pair $\left(N_{G}, \phi_{G}\right)$ to get a constraint for $\Delta_{N_{G}, \phi_{G}}$. This, together with the relation $\Delta_{N, \phi}^{\alpha}=\Delta_{N_{G}, \phi_{G}}$ proven in [FV08a, Lemma 3.3] and some straightforward calculations, shows that $\Delta_{N, \phi}^{\alpha}$ is monic of the degree stated.

Note that the adjunction inequality implies that $\zeta \cdot \phi \leq\|\phi\|_{T}$. If we had a way to show that this is an equality, then all twisted Alexander polynomials would have maximal degree. Theorem 3.1 would then suffice to show that $\phi \in H^{1}(N)$ is fibered. However (except in the case of $S^{1} \times N$ where we can use Kronheimer's theorem, see [Kr99] and [FV08a]) we are not aware of any direct way to show that $\zeta \cdot \phi=\|\phi\|_{T}$, which compels us to use the stronger Theorem 1.1.

\subsection{Proof of Theorem 1.3}

For the reader's convenience we recall the statement of Theorem 1.3.

Theorem 6.4. Let $N$ be a closed 3-manifold and let $p: M \rightarrow N$ be an $S^{1}$-bundle over $N$. Denote by $p_{*}: H^{2}(M ; \mathbb{R}) \rightarrow H^{1}(N ; \mathbb{R})$ the map which is given by integration along the fiber. Let $\psi \in H^{2}(M ; \mathbb{R})$. The following are equivalent:

(1) $\psi$ can be represented by a symplectic structure,

(2) $\psi$ can be represented by a symplectic structure which is $S^{1}$-invariant,

(3) $\psi^{2}>0$ and $\phi=p_{*}(\psi) \in H^{1}(N ; \mathbb{R})$ lies in the open cone on a fibered face of the Thurston norm ball of $N$.

Proof. The statement '(2) implies (1)' is trivial and the statement '(3) implies (2)' is proved in [FV10b], generalizing earlier work of Thurston [Th76], Bouyakoub [Bo88] and Fernández, Gray and Morgan [FGM91].

We now turn to the proof of '(1) implies (3)'. If $\psi \in H^{2}(M ; \mathbb{R})$ can be represented by a symplectic structure then it follows from the definition of being symplectic that $\psi^{2}>0$. 
Note that by [Th86, Theorem 5] a class $\phi \in H^{1}(N ; \mathbb{R})$ lies in the open cone on a fibered face of the Thurston norm ball of $N$ if and only if $\phi$ can be represented by a nondegenerate closed 1-form. It now follows from [FV10b, Proposition 3.1] that it suffices to prove '(1) implies (3)' for classes $\psi$ such that $p_{*}([\omega])$ is an integral class.

We first assume that the Euler class of the $S^{1}$-bundle is nontorsion. Suppose that we have $\psi \in H^{2}(M ; \mathbb{R})$ which can be represented by a symplectic structure such that $p_{*} \psi \in H^{1}(N)$ is an integral class. It follows immediately from Proposition 6.3 that $\Delta_{N, p_{*}(\psi)}^{\alpha}$ is nonzero for any epimorphism $\alpha: \pi_{1}(N) \rightarrow G$ onto a finite group. Therefore, as a consequence of Theorem 1.1 it follows that $p_{*}(\psi)$ is a fibered class.

We now assume that the Euler class of the $S^{1}$-bundle is trivial, i.e. $M=S^{1} \times N$. This case has been completely solved in [FV11a]. (More in the spirit of the present paper one can follow the argument for the case of nontorsion Euler class, replacing Proposition 6.3 by [FV08a, Proposition 4.4].)

Finally, if the Euler class of the $S^{1}$-bundle is torsion, then it is well known that there exists a finite cover of $N$ such that the pull-back $S^{1}$-bundle has trivial Euler class. We refer to [Bow09, Proposition 3] and [FV11c, Theorem 2.2] for details. Note that it is a consequence of Stallings' fibering theorem (see [St62]) that an integral class $\phi \in H^{1}(N ; \mathbb{Z})$ is fibered if and only if the pull back to a finite cover is fibered. The case of torsion Euler class now follows easily from this observation and from the product case. We leave the details to the reader.

Acknowledgments. We wish to thank Henry Wilton for very helpful conversations and we are grateful to the referee for thoughtful comments.

S. Vidussi was partially supported by NSF grant \#0906281.

\section{References}

[Ag08] Agol, I.: Criteria for virtual fibering. J. Topology 1, 269-284 (2008) Zbl 114857023 MR 2399130

[Ag12] Agol, I.: The virtual Haken conjecture (with an appendix by I. Agol, D. Groves, J. Manning). arXiv:1204.2810 (2012)

[AFW12] Aschenbrenner, M., Friedl, S., Wilton, H.: 3-manifold groups. arXiv:1205.0202 (2012)

[Ba01] Baldridge, S.: Seiberg-Witten invariants of 4-manifolds with free circle actions. Comm. Contemp. Math. 3, 341-353 (2001) Zbl 0987.57013 MR 1849644

[Ba03] Baldridge, S.: Seiberg-Witten invariants, orbifolds, and circle actions. Trans. Amer. Math. Soc. 355, 1669-1697 (2003) Zbl 1013.57019 MR 1946410

[Bon86] Bonahon, F.: Bouts des variétés hyperboliques de dimension 3. Ann. of Math. (2) 124, 71-158 (1986) Zbl 0671.57008 MR 0847953

[Bo88] Bouyakoub, A.: Sur les fibrés principaux de dimension 4, en tores, munis de structures symplectiques invariantes et leurs structures complexes. C. R. Acad. Sci. Paris Sér. I Math. 306 (1988), 417-420 Zbl 0635.53019 MR 0934608

[Bow09] Bowden, J.: The topology of symplectic circle bundles. Trans. Amer. Math. Soc. 361, 5457-5468 (2009) Zbl 1195.57059 MR 2515819

[Bow12] Bowden, J.: Symplectic 4-manifolds with fixed point free circle actions. arXiv:1206.0458 (2012) 
[Ch03] Cha, J.: Fibred knots and twisted Alexander invariants. Trans. Amer. Math. Soc. 355, 4187-4200 (2003) Zbl 102857004 MR 1990582

[EN85] Eisenbud, D., Neumann, W.: Three-Dimensional Link Theory and Invariants of Plane Curve Singularities. Ann. of Math. Stud. 110, Princeton Univ. Press, Princeton, NJ (1985) Zbl 0628.57002 MR 1990582

[FGM91] Fernández, M., Gray, A., Morgan, J. W.: Compact symplectic manifolds with free circle actions, and Massey products. Michigan Math. J. 38, 271-283 (1991) Zbl 0726.53028 MR 1098863

[FK06] Friedl, S., Kim, T.: The Thurston norm, fibered manifolds and twisted Alexander polynomials. Topology 45, 929-953 (2006) Zbl 1105.57009 MR 2263219

[FV08a] Friedl, S., Vidussi, S.: Twisted Alexander polynomials and symplectic structures. Amer. J. Math. 130, 455-484 (2008) Zbl 1154.57021 MR 2405164

[FV08b] Friedl, S., Vidussi, S.: Symplectic $S^{1} \times N^{3}$, surface subgroup separability, and vanishing Thurston norm. J. Amer. Math. Soc. 21, 597-610 (2008) Zbl 1142.57014 MR 2373361

[FV08c] Friedl, S., Vidussi, S.: Symplectic 4-manifolds with a free circle action. arXiv:0801. 1513 (2008)

[FV10a] Friedl, S., Vidussi, S.: A survey of twisted Alexander polynomials. In: The Mathematics of Knots, M. Banagl and D. Vogel (eds.), Contrib. Math. Comput. Sci. 1, Springer, 4594 (2011) Zbl 1223.57012 MR 2777847

[FV10b] Friedl, S., Vidussi, S.: Construction of symplectic structures on 4-manifolds with a free circle action. Proc. Roy. Soc. Edinburgh Sect. A 142, 359-370 (2012) MR 2911171

[FV11a] Friedl, S., Vidussi, S.: Twisted Alexander polynomials detect fibered 3-manifolds. Ann. of Math. 173, 1587-1643 (2011) Zbl 1231.57020 MR 2800721

[FV11b] Friedl, S., Vidussi, S.: Twisted Alexander polynomials and fibered 3-manifolds. In: Low-Dimensional and Symplectic Topology, Proc. Sympos. Pure Math. 82, Amer. Math. Soc., 111-130 (2011) Zbl 1244.57024 MR 2768657

[FV11c] Friedl, S., Vidussi, S.: Symplectic 4-manifolds with $K=0$ and the Lubotzky alternative. Math. Res. Lett. 18, 513-519 (2011) Zbl 1251.57018 MR 2802584

[GKM05] Goda, H., Kitano, T., Morifuji, T.: Reidemeister torsion, twisted Alexander polynomial and fibred knots. Comment. Math. Helv. 80, 51-61 (2005) Zbl 1066.57008 MR 2130565

[GP05] Goda, H., Pajitnov, A.: Twisted Novikov homology and circle-valued Morse theory for knots and links. Osaka J. Math. 42, 557-572 (2005) Zbl 1083.57017 MR 2166722

[Ham01] Hamilton, E.: Abelian subgroup separability of Haken 3-manifolds and closed hyperbolic $n$-orbifolds. Proc. London Math. Soc. (3) 83, 626-646 (2001) Zbl 1025.57007 MR 1851085

[JS79] Jaco, W., Shalen, P.: Seifert fibered spaces in 3-manifolds. Mem. Amer. Math. Soc. 21, no. 220 (1979) Zbl 0415.57005 MR 0539411

[Jo79] Johannson, K.: Homotopy Equivalences of 3-Manifolds with Boundaries. Lecture Notes in Math. 761, Springer, Berlin (1979) Zbl 0412.57007 MR 0551744

[KL99] Kirk, P., Livingston, C.: Twisted Alexander invariants, Reidemeister torsion and Casson-Gordon invariants. Topology 38, 635-661 (1999) Zbl 0928.57005 MR 1670420

[KM05] Kitano, T., Morifuji, T.: Divisibility of twisted Alexander polynomials and fibered knots. Ann. Scuola Norm. Sup. Pisa Cl. Sci. (5) 4, 179-186 (2005) Zbl 1117.57004 MR 2165406

[Kiy07] Kitayama, T.: Normalization of twisted Alexander invariants. arXiv:0705.2371 (2007) 
[Kr99] Kronheimer, P.: Minimal genus in $S^{1} \times M^{3}$. Invent. Math. 1351, 45-61 (1999) Zbl 0917.57017 MR 1664695

[La02] Lang, S.: Algebra. Rev. 3rd ed., Grad. Texts in Math. 211, Springer, New York (2002) Zbl 0984.00001

[LL95] Li, T. J., Liu, A. K.: Symplectic structures on ruled surfaces and generalized adjunction formula. Math. Res. Lett. 2, 453-471 (1995) MR 1355707

[Li01] Lin, X. S.: Representations of knot groups and twisted Alexander polynomials. Acta Math. Sinica (English Ser.) 17, 361-380 (2001) Zbl 0986.57003 MR 1852950

[Liu96] Liu, A. K.: Some new applications of general wall crossing formula, Gompf's conjecture and its cpplications. Math. Res. Lett. 3, 569-585 (1996) Zbl 0872.57025 MR 1418572

[MMP10] Manning, J. F., Martínez-Pedroza, E.: Separation of relatively quasiconvex subgroups. Pacific J. Math. 244, 309-334 (2010) Zbl 1201.20024 MR 2587434

[McC01] McCarthy, J.: On the asphericity of a symplectic $M^{3} \times S^{1}$. Proc. Amer. Math. Soc. 129, 257-264 (2001) Zbl 097253045 MR 1707526

[MT96] Meng, G., Taubes, C. H.: SW = Milnor torsion. Math. Res. Lett. 3, 661-674 (1996) Zbl 0870.57018 MR 1418579

[Pa07] Pajitnov, A.: Novikov homology, twisted Alexander polynomials, and Thurston cones. St. Petersburg Math. J. 18, 809-835 (2007) Zbl 1137.57017 MR 2301045

[Pa10] Pajitnov, A.: On the tunnel number and the Morse-Novikov number of knots. Algebr. Geom. Topol. 10, 627-635 (2010) Zbl 1196.57008 MR 2606794

[PRW02] Pajitnov, A. V., Rudolph, L., Weber, C.: Morse-Novikov number for knots and links. St. Petersburg Math. J. 13, 417-426 (2002) Zbl 1006.57003 MR 1850189

[PW11] Przytycki, P., Wise, D.: Graph manifolds with boundary are virtually special. arXiv: 1110.3513 (2011)

[Sc78] Scott, P.: Subgroups of surface groups are almost geometric. J. London Math. Soc. (2) 17, 555-565 (1978) Zbl 0412.57006 MR 0811778

[SW09a] Silver, D., Williams, S.: Nonfibered knots and representation shifts. In: Algebraic Topology-Old and New, Banach Center Publ. 85, Inst. Math., Polish. Acad. Sci., 101107 (2009) Zbl 1187.57010 MR 2503521

[SW09b] Silver, D., Williams, S.: Twisted Alexander polynomials and representation shifts. Bull. London Math. Soc. 41, 535-540 (2009) Zbl 1191.57010 MR 2506837

[St62] Stallings, J.: On fibering certain 3-manifolds. In: Topology of 3-Manifolds and Related Topics (1961), Prentice-Hall, Englewood Cliffs, NJ, 95-100 (1962) Zbl 1246.57049 MR 0158375

[Ta94] Taubes, C. H.: The Seiberg-Witten invariants and symplectic forms. Math. Res. Lett. 1, 809-822 (1994) Zbl 0853.57019 MR 1306023

[Ta95] Taubes, C. H.: More constraints on symplectic forms from Seiberg-Witten invariants. Math. Res. Lett. 2, 9-13 (1995) Zbl 0854.57019 MR 1312973

[Th76] Thurston, W. P.: Some simple examples of symplectic manifolds. Proc. Amer. Math. Soc. 55, 467-468 (1976) Zbl 0324.53031 MR 0402764

[Th86] Thurston, W. P.: A norm for the homology of 3-manifolds. Mem. Amer. Math. Soc. 59, no. 339, 99-130 (1986) Zbl 0585.57006 MR 0823443

[Tu01] Turaev, V.: Introduction to Combinatorial Torsions. Birkhäuser, Basel (2001) Zbl 0970.57001 MR 1809561

[Wa94] Wada, M.: Twisted Alexander polynomial for finitely presentable groups. Topology 33, 241-256 (1994) Zbl 082257006 MR 1273784

[WZ10] Wilton, H., Zalesskii, P.: Profinite properties of graph manifolds. Geom. Dedicata 147, 29-45 (2010) Zbl 1204.57019 MR 2660565 
[Wi09] Wise, D.: The structure of groups with a quasiconvex hierarchy. Electronic Res. Ann. Math. Sci. 16, 44-55 (2009) Zbl 1183.20043 MR 2558631

[Wi11] Wise, D.: From Riches to RAAGs: 3-Manifolds, Right-Angled Artin Groups, and Cubical Geometry. CBMS Reg. Conf. Ser. Math. 117, Amer. Math. Soc. (2012) Zbl pre06121354 MR 2986461

[Wi12] Wise, D.: The structure of groups with a quasiconvex hierarchy. Preprint (2012) from the webpage for the NSF-CBMS conference, $181 \mathrm{pp}$. 